\title{
Structural orientation dependent sub-lamellar bone mechanics
}

Ines Jimenez-Palomar ${ }^{1}$, Anna Shipov², Ron Shahar², Asa H Barber ${ }^{1,3}$

${ }^{1}$ Department of Materials, School of Engineering \& Materials Science, Queen Mary University of London, London E1 4NS, UK

${ }^{2}$ Koret School of Veterinary Medicine, The Hebrew University of Jerusalem, Israel

${ }^{3}$ School of Engineering, University of Portsmouth, Portsmouth P01 2UP, UK

\section{Corresponding Author}

asa.barber@port.ac.uk

+44(0)2392842363

\section{Keywords}

Bone, micromechanics, AFM, FIB

\section{Highlights}

A combination of SEM, AFM and FIB techniques were used to measure bone micromechanics.

The relationship between bone micromechanics and collagen orientation was studied. There is a direct relationship between elastic modulus and mineralised collagen orientation.

The results showed no correlation between elastic modulus with work-to-fracture.

\section{Abstract}

The lamellar unit is a critical component in defining the overall mechanical properties of bone. In this paper, micro-beams of bone with dimensions comparable to the lamellar unit were fabricated using focused ion beam (FIB) microscopy and mechanically tested in bending to failure using atomic force microscopy (AFM). A variation in the mechanical properties, including elastic modulus, strength and work to fracture of the micro-beams was observed and related to the collagen fibril orientation inferred from back-scattered scanning electron microscopy (SEM) imaging. Established mechanical models were further applied to describe the relationship between collagen fibril orientation and mechanical 
behaviour of the lamellar unit. Our results highlight the ability to measure mechanical properties of discrete bone volumes directly and correlate with structural orientation of collagen fibrils.

\section{Introduction}

Bone is a natural composite material and possesses a structural complexity across a range of length scales. This structural complexity is commonly based on the organizations of fibrils within lamellar units that allow bone to maintain biological function while performing a number of mechanical roles (Fratzl and Weinkamer, 2007; Wainwright et al., 1982). The structural organizations found at various length scales make the determination of bone mechanics challenging. Of all the structural features, Thus, the mechanical properties of the lamellar unit are critical in defining overall bone mechanics. Determining mechanical properties of lamellae is compromised by the micron length scale of the unit as well as the anisotropy of the constituents including the collagen predominantly in the form of fibrillar framework, hydroxyapatite platelets, non-collagenous protein and water (Gupta et al., 2006). Approaches to understand mechanics of the lamellar unit therefore consider a three phase composite material with plate-like hydroxyapatite minerals reinforcing collagen fibrils bound together in a relatively small volume fraction of non-collagenous proteins (Akiva et al., 1998). These hydroxyapatite minerals are platelet-shaped and embedded within and around the collagen fibrils, with the principal axis of the mineral oriented in the same direction as the long axis of the collagen fibrils (Fratzl et al., 2004; Landis et al., 1996; Wagermaier et al., 2007; Wagner and Weiner, 1992). Thus, the 
organization of the collagen fibrils within the lamellar unit defines the mineral orientation within this same unit. The orientation of collagen fibrils and, thus, the mineral phase in the lamellar unit has been the subject of considerable research due to the resultant influence on bone mechanics. Layered arrays of aligned mineralized collagen fibrils have been previously proposed to organize into five subunit layers within the lamellar unit (Weiner et al., 1997). The orientation of the sub layers has been shown to conform to a rotated plywood-like structure and can be generally grouped into two subunits (Boyd and Nigg, 2007; Gupta et al., 2006; Weiner et al., 1999); the 'thick' subunit where the collagen fibrils run parallel or at $30^{\circ}$ to the long axis of bone, thus contributing significantly to the elastic modulus (Ascenzi et al., 1982), and the 'thin' subunit for fibrillar arrays oriented at $60^{\circ}, 90^{\circ}$ and $120^{\circ}$ to the long axis. More recent work indicated considerably more disorder when examining bone volumes using 3D imaging due to fibril dispersions, suggesting subunit layers are better described using three sub-lamellar structures (Reznikov et al., 2013).

The overall mechanical properties of bone have shown to depend on both the volume fraction of constituents, most notably mineral phase, and the organization of these constituents represented by the lamellar unit. Previous works have indicated the importance of the mineral phase in defining overall bone mechanical behaviour by direct investigations on mineral volume fraction in a variety of different bone samples (Currey, 2006). A general increase in the elastic modulus of bone was correlated with an increase in the mineral volume fraction but a number of exceptions were noted where the mineral volume fraction alone does not determine the mechanical properties of bone. Currey suggested a mineral organizational factor that further defined the effectiveness of the reinforcement in bone, which has also been considered by Rho et al. (1998) (Rho et al., 
1998) and Sasaki et al. (1991) (Sasaki et al., 1991). The plywood collagen organisation within the lamellar unit has also been shown to act as a crack blunter to enhance toughening mechanisms at this submicrostructural level (Gupta et al., 2006; Peterlik et al., 2006). The mechanical properties of bone material are therefore not determined solely by mineral volume fraction but by both the mineral content and the mineral platelet orientation defined by the collagen fibril orientation (Sasaki et al., 1991). This mineralized fibril orientation will therefore give rise to the overall mechanical properties in bone material.

The influence of constituent organization on bone mechanical behaviour was first conclusively highlighted in studies on individual osteons. Polarized light microscopy was previously used to identify collagen fibril orientation and related to the mechanical properties of individual osteons in tension (Ascenzi et al., 1982), bending (Ascenzi and Bonucci, 1990) and compression (Ascenzi and Bonucci, 1968). Increases in the elastic modulus of individual osteons were found to occur when the majority of the collagen fibrils were oriented in the loading direction and supported theories that established the existence of lamellar orientations in bone material (Ascenzi et al., 1965; Giraud-Guille, 1988). Further works have more specifically highlighted the relationship between overall bone mechanics and collagen fibril orientation, including increased tensile strength (Martin and Ishida, 1989) and higher elastic modulus (Ramasamy and Akkus, 2007; Riggs et al., 1993) when collagen fibrils are predominantly oriented along the longitudinal, as opposed to the transverse, loading direction. The importance of the collagen fibril orientation in determining overall bone mechanical properties led to mechanical testing at smaller submicrostructural length scales. In particular, direct mechanical testing of bone at sub- 
millimetre length scales has been previously achieved in order to determine the effect of lamellar orientation on the elastic properties of baboon tibiae (Liu et al., 1999a). The results of the work by Liu et al. (1999) indicated clear anisotropic behaviour at different spatial positions along a bone fracture surface, and correlated lamellae orientation from mechanical behaviour and observed fracture surfaces. Further improvements to measure the mechanical properties of bone at the submicrostructural level have been attained through nanoindentation, which allows localized testing to be performed on individual constituents such as individual lamellae (Gupta et al., 2006; Lewis and Nyman, 2008; Xu et al., 2003). However, structural heterogeneities in bone coupled with the complex stress analysis formed from indentation of bone surfaces make direct understanding of bone component mechanics particularly fraught (Gupta et al., 2006; Isaksson et al., 2010; Rodriguez-Florez et al., 2013; Xu et al., 2003). A comprehensive review of nanoindentaton in mineralized tissue particularly emphasizes issues of indentation-sample contact area, critical in determining mechanical properties of samples, as an unexplored area of study ( Lewis and Nyman, 2008). A more recent study on nanoindentation as a technique to test the mechanical properties of bone has revealed the strong effect of the hydration state, tip geometry and the assumptions of the analysis methods on the results (Rodriguez-Florez et al., 2013) that provides discrepancies in the mechanical properties of bone measured via nanoindentation. Further determination of mechanical properties of constituents has been more recently available with the advent of more sophisticated and higher force resolution techniques, which are able to elucidate constituent mechanics directly. Such components have been investigated by a series of experiments such as nanoindentation (Tai et al., 2007), tensile testing of individual mineralized collagen fibrils (Hang and Barber, 2011) 
and collagen fibril pullout to determine the mechanical properties of non-collageneous protein regions (Hang et al. 2014).

Variations in the mechanical properties of bone when testing at different orientations to the bone's long axis are considered to be mainly due to the alignment of the collagen fibrils and mineral plates relative to the loading axis, and highlight the influence of the lamellar unit on overall bone mechanical behaviour (Fratzl et al., 2004; Martin and Ishida, 1989; Ramasamy and Akkus, 2007; Riggs et al., 1993). Techniques suitable for elucidating mechanical anisotropy defined by lamellae include nanoindentation (Gupta et al., 2006; Franzoso and Zysset, 2009), scanning acoustic microscopy (Granke et al., 2013) and micropillar compression (Schwiedrzik et al., 2014), with this latter work presenting one of the few data sets that examine failure beyond elastic limits. Computational validation has additionally stressed the importance of anisotropy on overall mechanical properties of bone (Geers et al., 2010; Ghanbari and Naghdabadi, 2009). Understanding the mechanical properties of the lamellar unit and the effects of orientation within the lamellae therefore provides a link between constituent and overall bone mechanical performance. While mechanical properties of constituents are instructive in defining overall bone behaviour, testing of bone at larger length scales approaching a few microns perhaps best represents the synergy between the constituents in bone but ignores the higher order structural effects such as osteonal canals or the curvature of whole bone. However, the synergy between the individual bone constituents even at these relatively small length scales is poorly understood. This paper attempts to evaluate the mechanics of the sub-lamellar unit through the study of tensile failure of rat femora. Considering that the width of a lamellar unit of rat bone is above $3 \mu \mathrm{m}$ (Akiva et al., 1998; Weiner et al., 1997; Reznikov et al., 2013), 
mechanical testing on discrete units of bone below this length scale is attempted. These sub-lamellar units will provide understanding of bone mechanics as a composite material both through consideration of the bone components and the organization of these components within the lamellar unit.

\section{Methods}

\section{Sample preparation}

The mechanical properties of individual micron sized units of bone were evaluated by first isolating individual bone beams from the parent bone material using a multi-stage process as carried out previously (Jimenez-Palomar et al., 2012). Briefly, the diaphysis of 8 month old sprague dawley rat femora were extracted and sectioned across their long length using a water-cooled diamond blade slow speed circular saw (Buehler, U.S.A). Rectangular samples of bone with dimensions of $12 \times 1 \times 1 \mathrm{~mm}$ were produced and stored in $70 \%$ ethanol. Bone samples were further dehydrated by submerging in a series of water/ethanol solutions with progressively increasing ethanol content. The dehydrated bone was gold coated and fixed to the sample stage using a two-part epoxy glue (Poxipol, Arg.). Isolation of discrete sub-lamellar units was achieved using focussed ion beam (FIB) milling according to previous work (Jimenez-Palomar et al., 2012) in a dual beam microscope (Quanta 3D, FEI, U.S.A./E.U.) incorporating both a scanning electron microscope (SEM) and FIB. We note that the smaller ion beam currents used avoid observable ion beam damage and FIB milling is always performed parallel to the sample faces, which reduce embedding gallium ions from the FIB within the discrete beam volumes produced. Resultant bone 
beams with approximate dimensions of $\sim 10 \times 2 \times 2 \mu \mathrm{m}$ were fabricated at regular intervals along the bone surface. Finally, an ion beam-milling step at a low current of $0.1 \mathrm{nA}$ was used to polish the surface of the bone beam samples and reveal collagen fibril organization in the bone structure using SEM back-scattered electron imaging as shown in Figure 1. We note the additional observation of porosity due to the canalicular network extending throughout the bone material (Reznikov et al., 2013). The network is reasonably periodic throughout and between the micro-beams as shown in Figure 1, which indicates a similarity of porosity within the micro-beams that are subsequently mechanically tested.

Samples were rehydrated prior to mechanical testing by removal from the dual beam chamber followed by placement in a closed vessel containing a high vapour concentration of Hank's buffer solution for two hours. This method of rehydration has been shown to be effective in maintaining FIB milled micro-beams wet in air but also in the vacuum conditions of an SEM (Jimenez-Palomar et al., 2012).

\section{Mechanical testing of bone micro-beams}

Mechanical testing of individual bone micro-beams were performed using a custom built atomic force microscope (AFM) (Attocube GmbH, Ger.) setup incorporated within the dual beam chamber (Hang et al., 2011). AFM cantilevers (AppNano, USA) with a spring constant of $28 \mathrm{Nm}^{-1}$ were used in this work. Mechanical testing was achieved by moving a FIB flattened AFM tip into contact with an individual bone micro-beam inside the SEM high 
vacuum $\left(5.25 \times 10^{-4} \mathrm{~Pa}\right)$ chamber of the dual beam system. The mechanical test can therefore be carefully monitored and recorded in situ via SEM so that the AFM tip contact with the bone beam is accurately known as shown in Figure 2a. FIB was used to flatten the apex of the AFM tip prior to mechanical testing to avoid AFM tip indentation into the sample. The force applied by the AFM causes a corresponding deflection in the bone beam during the mechanical bending tests. This force applied by the AFM tip is determined from an interferometer system recording the bending of the AFM cantilever, which is attached to the AFM tip in contact with the bone beam. The bending of the AFM cantilever can be translated to force by the formula $F=x k$ where $F$ is the applied force, $x$ is the AFM cantilever bending deflection recorded by the interferometer and $k$ is the spring constant of the cantilever, which is determined by the Sader calibration method (Ohler, 2007; Sader et al., 1995). Six individual bone micro-beams were fabricated and mechanically tested using the AFM system at a tip displacement rate of $0.04 \mu \mathrm{m} . \mathrm{s}^{-1}$. Elastic properties and fracture behaviour for individual beams were found using small deflection and large deflection, respectively, beam bending using the AFM setup as used previously (Jimenez-Palomar et al., 2012). Bending micro-beams to small deflections was achieved by applying a bone micro-beam displacement of up to $1.7 \mu \mathrm{m}$ whereas larger deflections beyond $3 \mu \mathrm{m}$ were required for sample failure, which was confirmed using the SEM observations with a $25^{\circ}$ sample tilt relative to the incident SEM beam as shown in Figure $2 \mathrm{~b}$. Testing of the bone beams within the SEM chamber was carried out within a time-frame of 2 hours. Previous work has indicated that micro-beams of bone remain hydrated within the vacuum chamber of a SEM within the testing time-frame of this work (Jimenez-Palomar et al., 2012). 
Simulation of the deformation of bone micro-beams was carried out using finite element analysis (FEA, FEA, Abaqus 6.7, Dassault Systèmes Simulia Corp., USA). A rectangular beam with dimensions of $2 \times 2 \times 10$ units was modelled. One beam end surface was anchored leaving all other surfaces free. A force was applied to the opposite end of the anchored surface to give resultant beam deformation. In order to simulate bone material, the beam was selected to have an elastic modulus taken from the experimental bone measurements. A von Mises stress analysis was used to show the point of highest concentration of stresses in this simulation in order to determine the location where collagen fibrils significantly contributed to the beam bending behaviour.

\section{Results}

\section{Bending of bone micro-beams to failure}

The force-displacement curves for six beams tested to failure are shown in Figure 3. These curves exhibit variations in the amount of force that was needed to displace and fracture each beam due to expected structural variations from collagen orientation within the bone micro-beams (Peterlik et al., 2006). A relatively linear force-displacement relationship was observed during beam bending, indicating apparent elastic behaviour up to fracture. The gradient of the linear region $(d f / d \delta)$ of the force-displacement curves was used to calculate an effective elastic modulus of the rat bone beam, $E$, using Equation (1):

$$
E=\frac{12 l^{3}}{3 b h^{3}} \cdot \frac{f}{\delta} \quad \text { Equation (1) }
$$

Where $l, b$ and $h$ are the length from the base of the sample to testing contact point, breadth and height of the rat bone beam respectively. Typical geometric values of the micro-beam 
are $l=10 \mu \mathrm{m}, b=2 \mu \mathrm{m}$ and $h=2 \mu \mathrm{m}$. We note that the breadth and height of the beams are measured by SEM at their base where the stress in maximal as shown below.

The strength and work-to-fracture for the micro-beams tested to failure were calculated using a variation of the equation described by Liu et al. (1999) (Liu et al., 1999b) adjusted for rectangular cross-sectioned samples as opposed to their circular cross-sectioned samples. Specifically, the failure strength and nominal work-to-fracture was calculated using previous equations for beam bending experiments (Koester et al., 2008; Liu et al., 1999b) as follows:

$$
\begin{array}{ll}
\sigma_{\max }=\frac{6 f l}{b h^{2}} & \text { Equation (2) } \\
W=\frac{A}{2 b h} & \text { Equation (3) }
\end{array}
$$

Where $l, b$ and $h$ are the length from the base of the sample to testing contact point, breadth and height of the rat bone beam respectively, $\sigma_{\max }$ is the maximum stress calculated from Equations (2), $W$ is the work-to-fracture and $A$ is the area under the force-displacement curve. Table 1 shows the elastic modulus, strength and work-to-fracture, calculated using Equation (1), (2) and (3) respectively, for the bone micro-beams mechanically tested in bending to failure from the data shown in Figure 3. The error in mechanical property values were calculated from the standard deviation caused by small changes in AFM tip contact point along the length of the micro-beams during bending. Contact point changes 
arise from deflections of both the bone micro-beam and AFM cantilever and are observed by the SEM during mechanical testing.

\section{Discussion}

The mechanical properties of the micro-beams tested at various locations along the edge of the bone sample, such as shown in Figure 1, provide a range of values listed in Table 1. The elastic modulus measured from the bone micro-beam bending tests are comparable to values obtained from previous work but using different mechanical testing experiments (Gupta et al., 2006; Liu et al., 1999b). Collagen fibril orientation is expected to be dominant in determining the mechanical properties of each micro-beam due to the known change in the organization of fibrils throughout bone material. Elastic modulus is perhaps the clearest indication of collagen fibril orientation. In terms of proposing a model to describe fibril organization within a lamellar unit, a regular ordered 5 layered subunit structure composed of aligned mineralized collagen fibrils with an offset of around $30^{\circ}$ is used according to plywood-type behaviour (Weiner et al., 1997) as shown in Figure 4. We note that more accurate structural observations of lamellae have been made (Reznikov et al., 2013) but are more difficult to model due to the disorder of fibrils found.

Figure 5 shows the variation in elastic modulus of the bone micro-beams as a function of spatial location along the edge of the bone sample. The red curve was fitted to the elastic

modulus values using a sine curve function of $y=y_{0}+A \cdot \sin \left(2 \pi\left(x-x_{c}\right) / w\right)$, fixing an upper 
boundary $3.2 \mu \mathrm{m}$ periodicity of the lamellar unit of rat long bones (Weiner et al., 1997) using software (Origin Pro 8, OriginLab, USA). The fitting gave parameters of $y_{0}=7.06 \pm 0.82$, $\mathrm{A}=5.23 \pm 1.8, \mathrm{x}_{\mathrm{c}}=3.1 \pm 0.15$ and $\mathrm{w}=3.04 \pm 0.01$.

The measured changes in the elastic modulus of the bone micro-beams are expected to be due to the collagen fibril orientations within the beam. In particular, a micro-beam consisting predominantly of collagen aligned mainly along the long axis of the micro-beam will give a relatively large elastic modulus whereas a micro-beam composed of collagen oriented perpendicular to the long axis of bone provides a relatively low elastic modulus value. To validate this assumption, SEM back-scattered imaging was used to examine the FIB polished surfaces of the micro-beams. Back-scattered imaging provides atomic number contrast for relatively flat samples. Thus, orientation in mineralized collagen fibrils can be quantified using back-scattered imaging due to the relatively high atomic number mineral phase along the collagen fibril length. Figure 6 shows an individual bone micro-beam with a series of linear patterns observed from the base of the beam to the end of the beam. Linear patterns in bone material in this work show similarities to fibrillar patterns observed in transmission electron micrographs collected from demineralised lamellar rat femur and tibia samples (Weiner et al., 1997; Weiner et al., 1999). The linear patterns are expected to correspond to the mineral in the oriented collagen fibrils and are seen to change from the micro-beam base to end in Figure 6. We note that the linear banding patterns vary in direction along the length of the micro-beams and, thus, cannot be from the FIB preparation as this was carried out in one direction only. 
An overall fibril orientation in the micro-beam is potentially difficult to define as a number of different collagen fibril orientations exist within a lamellar unit and the micro-beam tested may bridge between multiple lamellae. However, an average mineralized fibril orientation in the micro-beams can be defined by measuring the linear pattern angle at 10 equidistant points along the length of the micro-beam as shown in Figure 6. Each orientation was measured three times at each point along the length, with all values the same to within less than $1^{\circ}$. A total of 5 beams were examined with the fibril orientation angle relative to the length of the micro-beam along the length of each beam. shown in Table 2.

FEA of an individual bone micro-beam under bending was carried out in order to determine the orientation along the micro-beam length that contributed significantly to the overall beam bending behaviour. The FEA experiment shown in Figure 7 highlights a concentration of stresses in the first $10 \%$ of the length of the beam, which indicates that the collagen fibril orientation within this first $10 \%$ of the beam length is expected to define bone micro-beam mechanics during bending tests.

The measured fibril/platelet orientation observed via the back-scattered SEM images as shown in Table 2 can be related to the elastic modulus of the bone micro-beams found from AFM mechanical testing. The elastic modulus of the bone micro-beams is considered to 
increase as the orientation angle decreases. Thus, collagen fibrils and platelets oriented along the long axis of the micro-beam (i.e. the $0^{\circ}$ angle) provide an increased elastic modulus whereas progressive off-axis alignment gives a drop in the micro-beam elastic modulus. Such observations of orientation at FIB polished micro-beam surfaces using SEM back-scattered imaging is potentially a method for characterizing structural orientation and resultant bone micro-beam mechanics. The micro-beam elastic modulus variation with orientation is therefore further described using a theoretical composite model such as achieved previously by Akiva et al. (1998) (Akiva et al., 1998). Using the structural parameters given by Akiva et al. (1998) (Akiva et al., 1998), the theoretical elastic modulus of a lamellar unit when all collagen fibrils are aligned along the long axis of bone and when the collagen fibrils are aligned perpendicular to the bone long axis can be calculated using Equations (4) and (5) respectively.

$$
\begin{array}{ll}
E_{1}=E_{m}\left(1-V_{p}\right)+E_{p} V_{p} & \text { Equation (4) } \\
E_{2}=E_{3}=\left[\frac{V_{p}}{E_{p}}+\frac{\left(1-V_{p}\right)}{E_{m}}\right]^{-1} & \text { Equation (5) }
\end{array}
$$

Where $E_{1}$ is the theoretical elastic modulus of a lamellar unit with all of the platelet reinforced collagen fibrils aligned along the long axis of the bone, $E_{2}$ is the elastic modulus of a lamellar unit when these fibrils are all aligned transverse and perpendicular to the long axis of the bone and $E_{3}$ is the elastic modulus when the collagen fibrils are aligned transversely out-of-plane. $V_{p}$ is the platelet volume fraction taken as 0.5 (Akiva et al., 1998; Doty et al., 1976; Ziv et al., 1996), $E_{m}$ is the elastic modulus in tension of collagen taken as 2.4 $\pm 0.4 \mathrm{GPa}$ (Hang et al., 2011) and $E_{p}$ the elastic modulus of the mineral platelets taken as 
114 GPa (Akiva et al., 1998; Gilmore and Katz, 1982). The elastic modulus values calculated for a lamellar unit using Equations 4 and 5 give upper and lower limits of $E_{1}=58.2 \mathrm{GPa}$ and $E_{2}=E_{3}=4.7 \mathrm{GPa}$ respectively. Following the modelling by Akiva et al. (1998), these elastic modulus values were then related to collagen fibril orientation using:

$$
\begin{aligned}
& E(\theta, \varphi)=\frac{\cos ^{4} \theta}{E_{1}}+\frac{\sin ^{4} \theta \cos ^{4} \varphi}{E_{2}}+\frac{\sin ^{4} \theta \sin ^{4} \varphi}{E_{3}}+\cos ^{2} \theta \sin ^{2} \theta \cos ^{2} \varphi I_{12} \\
& +\sin ^{4} \theta \sin ^{2} \varphi \cos ^{2} \varphi I_{23}+\sin ^{2} \theta \cos ^{2} \theta \sin ^{2} \varphi I_{31}
\end{aligned}
$$

Where

$$
I_{i j}=\frac{1}{G_{i j}}-2 \frac{v_{i j}}{E_{i j}}(i, j=1,2,3, i \neq j)
$$

Equation (7)

and $E$ is the elastic modulus of the micro-beam, $\theta$ is the collagen fibril orientation angle varying from $0^{\circ} \leq \theta \leq 90^{\circ}, \varphi$ is the angle of mechanical testing as known from the SEM observations, $G$ is the shear modulus of the micro-beam approximated by $G=E / 2(1+v)$ where $v$ is the Poisson's ratio with a value of 0.35 (Akiva et al., 1998) and $i j$ is the testing axis. The variation in micro-beam elastic modulus, previously shown in Figure 5, with the predominant collagen fibril orientation found from back-scattered imaging data in Table 1 is shown in Figure 8.

The fit between the variation of the elastic modulus of the bone micro-beams with fibril orientation using the relatively simple model of Equation (6) highlights how the elastic 
modulus can be approximated from orientation observations using the back-scattered SEM imaging. We can therefore state that the mechanical properties of the bone micro-beams are defined by fibril orientation and not, for example, by canaliculae. The AFM mechanical testing of the micro-beams provides further mechanical information based on failure including the strength and work-to-fracture, which may give additional orientation dependent variations as observed for elastic modulus. To examine these failure related mechanical properties, a plot of the micro-beam strength and work-to fracture with the variation in elastic modulus is presented in Figure 9 below.

General trend lines in Figure 9 highlights an increase in the bone micro-beam elastic modulus as the strength of the beam increases but little variation in work-to-fracture with respect to elastic modulus. The mechanical properties shown in Figure 9 can be compared to existing literature. Strength values from bending of rat whole bones are between $134 \pm 4$ MPa (Barengolts et al., 1993) to $180 \pm 6$ MPa (Jorgensen et al., 1991), which are similar to strengths from compression testing of samples with relatively small cross sectional areas of the order of $1 \mathrm{~mm}^{2}$ (Cory et al., 2010). Such measured strength values are considerably smaller than reported in this work and is expected to be because bone micro-beams are relatively dense, with an absence of voids that would compromise resultant strength found in larger sample volumes. Conversely, elastic modulus is not so dependent on defects and exhibits a similarity between our work and values ranging from $4.9 \pm 4 \mathrm{GPa}$ (Ejersted et al., 1993 ) to $8 \pm 0.4 \mathrm{GPa}$ (Barengolts et al., 1993) for whole bone and $5.12 \pm 0.77 \mathrm{GPa}$ (Kasra, et al., 1997) to $8.8 \pm 2.5$ GPa (Cory et al., 2010) for samples with millimeter scale widths. 
Previous nanoindentation experiments typically provide elastic modulus values significantly higher than the micron to millimeter length scales, with corresponding structural changes at the smaller length scales attributed to such variations (Cory et al., 2010). The effect of collagen fibril orientation on the strength of a bone micro-beam is similar to elastic modulus dependence, with collagen fibrils organized along the principal beam axis fracturing during failure whereas progressive off-axis fibril orientations promoting failure of the weaker interfaces between the fibrils. Indeed, the strength of the non-collagenous protein region has been shown to be many orders of magnitude lower than fibril strength (Hang et al. 2014). Thus, the mineralized collagen fibrils in the microbeams behave as fibrous reinforcements that provide strength and stiffness along the main orientation axis. However, the work-to-fracture of the micro-beams is observed to vary little with the elastic modulus of the micro-beam, which is opposed to typically composite design where off-axis reinforcing fibres allow enhanced crack-deflection between the reinforcement phase for improved toughness (Hull and Clyne, 2001). Maintaining a bone work-to-fracture that is independent of the mineralized collagen fibril orientation is therefore surprising and indicates that the failure at the base of the beam potentially fractures the same number of collagen fibrils and their corresponding interfaces. Homogeneous work-to-fracture at the micron length scales examined here is in contrast to the behaviour of bulk bone specimens where directional toughening is clearly known (Koester et al., 2008). Importantly, this previous work highlighted relatively large structural features such as bone cement lines that control crack propagation to enhance toughness in a 'breaking' direction but show significant lowering of toughness in the bone 'splitting' direction. The bone micro-beams examined here do not contain the larger scale 
cement line features as rat bone is unremodelled and would therefore demonstrate a lack of directional dependent toughness behaviour that originates from cement lines, thus emphasizing the control of structure over material properties in defining bone toughness. Additional defects from canaliculi have also been proposed as dictating micro-cracking mechanisms (Behiri and Bonfield, 1984; Evans, 1973) (Bonfield and Datta, 1976; Bonfield and $\mathrm{Li}, 1966)$ but are not considered in our current work. Our results must therefore be considered as evaluating the mechanical properties of the intrinsic sub-lamellar bone material to failure, which may be important when the quality of the bone material varies such as from the effects of disease.

\section{Conclusions}

Bone micro-beams with dimensions comparable to the lamellar unit were successfully isolated using focused ion beam (FIB) microscopy and mechanically tested in bending using atomic force microscopy (AFM). A variation in the mechanical properties of the micro-beams was observed and directly related to the mineralised collagen fibril orientation inferred from back-scattered scanning electron microscopy (SEM) imaging. Mechanical models were applied to describe the relationship between collagen fibril orientation and mechanical behaviour of the lamellar unit. The strength and elastic modulus of the bone micro-beams were defined by fibril orientation and conformed to fibrous composite considerations where reinforcing fibrils aligned in the direction of the load gave the highest strength and stiffness. Work-to-fracture exhibited orientation independence was suggested as being an intrinsic bone property from the sub-lamellar 
unit of our work that ignores larger structural features that provide orientation dependent toughness found in whole bone samples.

\section{Acknowledgements}

I. J.-P. thanks the EPSRC for scholarship funding. The authors thank Dr Ken Png and Mick Willis for help with sample preparation, the NanoVision Centre at Queen Mary University of London for use of facilities and Carlos J. Pasquali for developing Matlab and Octave scripts for AFM data analysis.

\section{References}

Akiva, U., Wagner, H.D., Weiner, S., 1998. Modelling the three-dimensional elastic constants of parallel-fibred and lamellar bone. Journal of Materials Science 33, 1497-1509.

Ascenzi, A., Benvenuti, A., Bonucci, E., 1982. The Tensile Properties of Single Osteonic Lamellae: Technical Problems and Preliminary Results. Journal of Biomechanics 15, 29-37. Ascenzi, A., Bonucci, E., 1968. The compressive properties of single osteons. Anatomical Record 161, 377-392.

Ascenzi, A., Bonucci, E., 1990. The bending properties of single osteons. Journal Biomechanics 23, 763-771.

Ascenzi, A., Bonucci, E., Boccarielli, D.S., 1965. An electron microscope study of osteon calcification. J. Ultrastruct Res 12, 287.

Barengolts, E.I., Curry, D.J., Bapna, M.S., Kukreja, S.C., 1993. Effects of Endurance Exercise on Bone Mass and Mechanical Properties in Intact and Ovariectomized Rats. Journal of Bone and Mineral Research 8, 937-942.

Behiri, J.C., Bonfield, W., 1984. Fracture Mechanics Of Bone-The Effects Of Density, Specimen Thickness And Crack Velocity On Longitudinal Fracture. Journal of Biomechanics 17, 25-34.

Bonfield, W., Datta, P.K., 1976. Fracture Toughness Of Compact Bone. Journal of Biomechanics 9, 131-134.

Bonfield, W., Li, C.H., 1966. Deformation and fracture of bone. J. Appl. Phys. 37, 869-875.

Boyd, S.K., Nigg, B.M., 2007. Bone, in: Nigg, B.M., Herzog, W. (Eds.), Biomechanics of the Musculo-skeletal System, 3rd ed ed. Wiley, Canada, pp. 65-94. 
Cory, E., Nazarian, A., Entezari, V., Vartanians, V., Müller, R., Snyder, B.D., 2010. Compressive axial mechanical properties of rat bone as functions of bone volume fraction, apparent density and micro-ct based mineral density. Journal of Biomechanics 43, 953-960.

Currey, J.D., 2006. Bones Structure and Mechanics, 2nd ed. Princeton University Press.

Doty, S., Robinson, R.A., Schofield, B., 1976. Morphology of bone and histochemical staining characteristics of bone cells, in: Aurbach, G.D. (Ed.), Handbook of Physiology. American Physiology Society, Washington D.C., pp. 3-23.

Ejersted, C., Andreassen, T.T., Oxlund, H., Jorgensen, P.H., Bak, B., Haggblad, J., Torring, O., Nilsson, M.H.L., 1993. Human Parathyroid Hormone (1-34) and (1-84) Increase the Mechanical Strength and Thickness of Cortical Bone in Rats. Journal of Bone and Mineral Research 8, 1097-1101.

Evans, F.G., 1973. Mechanical Properties of Bone. Charles C. Thomas, Springfield.

Franzoso, G., Zysset, P.K., 2009. Elastic Anisotropy of Human Cortical Bone Secondary Osteons Measured by Nanoindentation. Journal of Biomechanical Engineering 131, 021001. Fratzl, P., Gupta, H.S., Paschalis, E.P., Roschger, P., 2004. Structure and mechanical quality of the collagen-mineral nano-composite in bone. Journal of Materials Chemistry 14, 21152123.

Fratzl, P., Weinkamer, R., 2007. Nature's hierarchical materials. Progress in Materials Science 52, 1263-1334.

Geers, M.G.D., Kouznetosova, V.G., Brekelmans, W.A.M., 2010. Multi-scale computational homogenization: Trends and challenges. Journal of Computational and Applied Mathematics 234, 2175-2182.

Ghanbari, J., Naghdabadi, R., 2009. Nonlinear hierarchical multiscale modeling of cortical bone considering its nanoscale microstructure. Journal of Biomechanics 42, 1560-1565.

Gilmore, R.S., Katz, J.L., 1982. Elastic properties of apatites. Journal of Materials Science 17, 1131-1141.

Giraud-Guille, M.M., 1988. Twisted Plywood Architecture of Collagen Fibrils in Human Compact Bone Osteons. Calcified Tissue International 42, 167-180.

Granke, M., Gourrier, A., Rupin, F., Raum, K., Peyrin, F., Burghammer, M., Saïed, A., Laugier, P., 2013. Microfibril Orientation Dominates the Microelastic Properties of Human Bone Tissue at the Lamellar Length Scale. PloS One 8, 58043.

Gupta, H.S., Stachewicz, U., Wagermaier, W., 2006. Mechanical modulation at the lamellar level in osteonal bone. J. Mater. Res. 21, 1913-1921.

Hang, F., Barber, A.H., 2011. Nano-mechanical properties of individual mineralized collagen fibrils from bone tissue. J. R. Soc. Interface 8, 500-505.

Hang, F., Lu, D., Bailey, R.J., Jimenez-Palomar, I., Stachewicz, U., Cortes-Ballesteros, B., Davies, M., Zech, M., Bödefeld, C., Barber, A.H., 2011. In situ tensile testing of nanofibers by combining atomic force microscopy and scanning electron microscopy. Nanotechnology 22. Hang, F., Gupta, H.S., Barber, A.H., 2014. Nanointerfacial strength between non-collagenous protein and collagen fibrils in antler bone. J. R. Soc. Interface 11, 20130993.

Hull, D., Clyne, T.W., 2001. An Introduction to Composite Materials, 2nd ed ed. Cambridge University Press, Cambridge, UK.

Isaksson, H., Nagao, S., Malkiewicz, M., Julkunen, P., Nowak, R., Jurvelin, J.S., 2010. Precision of nanoindentation protocols for measurement of viscoelasticity in cortical and trabecular bone. Journal of Biomechanics 43, 2410-2417. 
Jimenez-Palomar, I., Shipov, A., Shahar, R., Barber, A.H., 2012. Influence of SEM vacuum on bone micromechanics using in situ AFM. Journal of the Mechanical Behavior of Biomedical Materials 5, 149-155.

Jorgensen, P.H., Bak, B., Andreassen, T.T., 1991. Mechanical Properties and Biochemical Composition of Rat Cortical Femur and Tibia after Long-Term Treatment with Biosynthetic Human Growth Hormone. Bone 12, 353-359.

Koester, K.J., Ager, J.W., Ritchie, R.O., 2008. The true toughness of human cortical bone measured with realistically short cracks. Nature Materials 7, 672-677.

Kasra, M., Vanin, C.M., MacLusky, N.J., Casper, R.F., Grynpas, M.D., 1997. Effects of Different Estrogen and Progestin Regimens on the Mechanical Properties of Rat Femur. Journal of Orthopaedic Research 15, 118-123.

Landis, W.J., Hodgens, K.J., Arena, J., Song, M.J., McEwen, B.F., 1996. Structural relations between collagen and mineral in bone as determined by high voltage electron microscopic tomography. Microscopy Research and Technique 33, 192-202.

Lewis, G., Nyman, J.S., 2008. The Use of Nanoindentation for Characterizing the Properties of Mineralized Hard Tissues: State-of-the Art Review. Journal of Biomedical Materials Research Part B: Applied Biomaterials 87B, 286-301.

Liu, D., Weiner, S., Wagner, D., 1999a. Anisotropic mechanical properties of lamellar bone using miniature cantilever bending specimens. Journal of Biomechanics 2, 647-654.

Liu, D., Weiner, S., Wagner, D., 1999b. Anisotropic mechanical properties of lamellar bone using miniature cantilever bending specimens. Journal of Biomechanics 2, 647\}654.

Martin, R.B., Ishida, J., 1989. The Relative Effects of Collagen Fiber Orientation, Porosity, Density and Mineralization on Bone Strength. Journal of Biomechanics 22, 419-426.

Ohler, B., 2007. Practical Advice on the Determination of Cantilever Spring Constants. Veeco Instruments Inc., Veeco Instruments Inc., pp. 1-12.

Peterlik, H., Roschger, P., Klaushofer, K., Fratzl, P., 2006. From Brittle To Ductile Fracture Of Bone. Nature Materials 5, 52-55.

Ramasamy, J.G., Akkus, O., 2007. Local variations in the micromechanical properties of mouse femur: The involvement of collagen fiber orientation and mineralization. Journal of Biomechanics 40, 910-818.

Reznikov, N., Almany-Magal, R., Shahar, R., Weiner, S., 2013. Three-dimensional imaging of collagen fibril organization in rat circumferential lamellar bone using a dual beam electron microscope reveals ordered and disordered sub-lamellar structures. Bone 52, 676-683.

Rho, J.Y., Kuhn-Spearing, L., Zioupos, P., 1998. Mechanical properties and the hierarchical structure of bone. Medical Engineering \& Physics 20, 92-102.

Riggs, C.M., Vaughan, L.C., Evans, G.P., Lanyon, L.E., Boyde, A., 1993. Mechanical implications of collagen fibre orientation in cortical bone of the equine radius. Anatomy and Embryology 187, 239-248.

Rodriguez-Florez, N., Oyen, M.L., Shefelbine, S.J., 2013. Insight into differences in nanoindentation properties of bone. Journal of the Mechanical Behavior of Biomedical Materials 18, 90-99.

Sader, J.E., Larson, I., Mulvaney, P., White, L.R., 1995. Method for the calibration of atomic force microscope cantilevers. Rev. Sci. Instrum. 66, 3789-3798.

Sasaki, N., Ikawa, T., Fukuda, A., 1991. Orientation of Mineral in bovine Bone and the Anisotropic Mechanical Properties of Plexiform Bone. Journal of Biomechanics 24, 57-61. 
Schwiedrzik, J., Raghavan, R., Bürki, A., LeNader, V., Wolfram, U., Michler, J., Zysset, P., 2014. In situ micropillar compression reveals superior strength and ductility but an absence of damage in lamellar bone. Nature Materials 13, 740-747.

Tai, K., Dao, M., Suresh, S., Palazoglu, A., Ortiz, C., 2007. Nanoscale heterogeneity promotes energy dissipation in bone. Nature Materials 6, 454-462.

Wagermaier, W., Gupta, H.S., Gourrier, A., Paris, O., Roschger, R., Burghammer, M., Riekel, C., Fratzl, P., 2007. Scannig texture analysis of lamellar bone using microbeam synchrotron $\mathrm{x}$ ray radiation. Applied Crystallography 40, 115-120.

Wagner, D., Weiner, S., 1992. On the relationship between the microstructure of bone and its mechanical stiffness. Journal of Biomechanics 25, 1311-1320.

Wainwright, S.A., Biggs, W.D., Currey, J.D., Gosline, J.M., 1982. Mechanical Design in Organisms. Princeton University Press.

Weiner, S., Arad, T., Sabanay, I., Traub, W., 1997. Rotated Plywood Structure of Primary Lamellar Bone in the Rat: Orientations of the Collagen Fibril Arrays. Bone 20, 509-514.

Weiner, S., Traub, W., Wagner, H.D., 1999. Lamellar Bone: Structure-Function Relations. Journal of Structural Biology 126, 241-255.

$\mathrm{Xu}$, J., Rho, J.Y., Mishra, S.R., Fan, Z., 2003. Atomic force miscroscopy and nanoindentation characterization of human lamellar bone prepared by microtome sectioning and mechanial polishing technique. Journal of Biomedical Materials Research 67A, 719-726.

Ziv, V., Wagner, H.D., Weiner, S., 1996. Microstructure-microhardness relations in parallelfibered and lamellar bone. Bone 18, 417-428.

\section{Figure and table captions}

Table 1. Work-to-fracture and elastic modulus values of rat bone femur micro-beams tested in bending to failure. 
Figure 1. Electron micrograph showing a back-scattered electron image of bone microbeam samples. Porosity due to the canalicular network is observed within the micro-beams as dark regions.

Figure 2. SEM micrographs showing a) in situ cantilever beam testing in bending provided by the AFM tip pushing into the free end of the bone micro-beam until b) failure of the micro-beam occurs.

Figure 3. AFM force-deflection curves for six sub-lamellar micro-beams tested to fracture in bending.

Figure 4. Schematic showing the approximate dimensions of the sub-lamellar unit in bone material produced using FIB techniques. The FIB milled bone micro-beam width is $2 \mu \mathrm{m}$, which is below the width of a single lamellar unit, thus indicating that the testing is sublamellar. In the diagram, the micro-beam contains part of the lamellar unit with the collagen fibril orientation predominantly along the long axis of the micro-beam, which will result in an elastic modulus maximum for the micro-beam.

Figure 5. Plot of the variation in bone micro-beam elastic modulus against beam position (top) at the edge of the bone sample shown in the SEM micrograph (bottom).

Figure 6. SEM micrograph of an individual FIB polished bone micro-beam with markers indicating the percentage distance along the beam where the fibril orientation angle was measured. Arrows highlight the observed orientation angle.

Figure 7. FEA modelling of a cantilever beam in bending, fixed at the right of the image, with a concentrated load applied to the beam free end at the left of the image. Red 
colouration corresponds to higher stresses while the blue corresponds to the lowest stresses.

Figure 8. Plot of the variation of bone micro-beam elastic modulus with collagen fibril orientation angle taken from SEM images as demonstrated in Figure 6. A fit line from Equation (6) is provided.

Figure 9. Plot of a) the strength of bone micro-beams against elastic modulus, showing a general increasing modulus with increasing strength $\left(R^{2}=0.6\right)$ and $\left.b\right)$ no discernable trend in work-to-fracture with increasing elastic modulus.

\section{Tables}

Table 1. Work-to-fracture and elastic modulus values of rat bone femur micro-beams tested in bending to failure.

\begin{tabular}{lll}
$\begin{array}{l}\text { Work-to-fracture } \\
\left(\mathrm{Jm}^{-2}\right)\end{array}$ & $\begin{array}{l}\text { Strength } \\
(\mathrm{MPa})\end{array}$ & $\begin{array}{l}\text { Elastic } \\
\text { Modulus }\end{array}$ \\
\hline
\end{tabular}




\section{(GPa)}

\begin{tabular}{lll}
\hline $24.8 \pm 3.7$ & $847 \pm 127$ & $8.29 \pm 1.24$ \\
\hline $27.2 \pm 4.1$ & $632 \pm 95$ & $4.98 \pm 0.75$ \\
\hline $31.2 \pm 4.7$ & $952 \pm 143$ & $6.03 \pm 0.91$ \\
\hline $35.4 \pm 5.3$ & $1083 \pm 162$ & $11.24 \pm 1.69$ \\
\hline $36.7 \pm 5.5$ & $797 \pm 120$ & $6.15 \pm 0.92$ \\
\hline $40.6 \pm 6.1$ & $750 \pm 113$ & $3.68 \pm 0.55$ \\
\hline
\end{tabular}

Table 2. Comparison of the elastic modulus of the FIB milled bone micro-beams with the fibril angle in degrees of the orientation along the length of the micro-beam, as indicated by the percentage of the total micro-beam length from the base. 
Beam 1 Beam 2 Beam 3 Beam 4 Beam 5 Beam 6*

\begin{tabular}{|c|c|c|c|c|c|c|}
\hline $\begin{array}{l}\text { Modulus } \\
\text { (GPa) }\end{array}$ & $4.98 \pm 0.25$ & $8.29 \pm 0.30$ & $11.24 \pm 0.20$ & $6.15 \pm 0.19$ & $3.68 \pm 0.18^{1}$ & $6.03 \pm 0.3^{1}$ \\
\hline \multicolumn{7}{|l|}{$\begin{array}{l}\text { Percentage } \\
\text { from base }\end{array}$} \\
\hline $10 \%$ & $\mathrm{~N} / \mathrm{A}$ & $41_{-}^{\circ}$ & $32_{-}^{\circ}$ & $66_{-}^{\circ}$ & $68_{-}^{\circ}$ & $43_{-}^{\circ}$ \\
\hline $20 \%$ & & $54_{-}^{\circ}$ & $40_{-}^{\circ}$ & $90_{-}^{\circ}$ & $65_{-}^{\circ}$ & $44_{-}^{\circ}$ \\
\hline $30 \%$ & & $47_{-}^{\circ}$ & $42_{-}^{\circ}$ & $63_{-}^{\circ}$ & $64_{-}^{\circ}$ & $46_{-}^{\circ}$ \\
\hline $40 \%$ & & $36_{-}^{\circ}$ & $34_{-}^{\circ}$ & $71_{-}^{\circ}$ & $63^{\circ}$ & $35_{-}^{\circ}$ \\
\hline $50 \%$ & & $25_{-}^{\circ}$ & $49_{-}^{\circ}$ & $61_{-}^{\circ}$ & $88_{-}^{\circ}$ & $43_{-}^{\circ}$ \\
\hline $60 \%$ & & $35_{-}^{\circ}$ & $38_{-}^{\circ}$ & $65_{-}^{\circ}$ & $37_{-}^{\circ}$ & $46_{-}^{\circ}$ \\
\hline $70 \%$ & & $33_{-}^{\circ}$ & $40_{-}^{\circ}$ & $75^{\circ}$ & $46^{\circ}$ & $20_{-}^{\circ}$ \\
\hline $80 \%$ & & $47_{-}^{\circ}$ & $27_{-}^{\circ}$ & $90_{-}^{\circ}$ & $\mathrm{N} / \mathrm{A}$ & $23_{-}^{\circ}$ \\
\hline $90 \%$ & & $44_{-}^{\circ}$ & $37_{-}^{\circ}$ & $76_{-}^{\circ}$ & $\mathrm{N} / \mathrm{A}$ & $6_{-}^{\circ}$ \\
\hline $100 \%$ & & $\mathrm{~N} / \mathrm{A}$ & $\mathrm{N} / \mathrm{A}$ & $\mathrm{N} / \mathrm{A}$ & $\mathrm{N} / \mathrm{A}$ & $\mathrm{N} / \mathrm{A}$ \\
\hline $\begin{array}{l}\text { Total } \\
\text { average } \\
\text { erientation }\end{array}$ & & $40_{-}^{\circ}$ & $38_{-}^{\circ}$ & $73^{\circ}$ & $62_{-}^{\circ}$ & $34_{-}^{\circ}$ \\
\hline
\end{tabular}




\section{Figures}

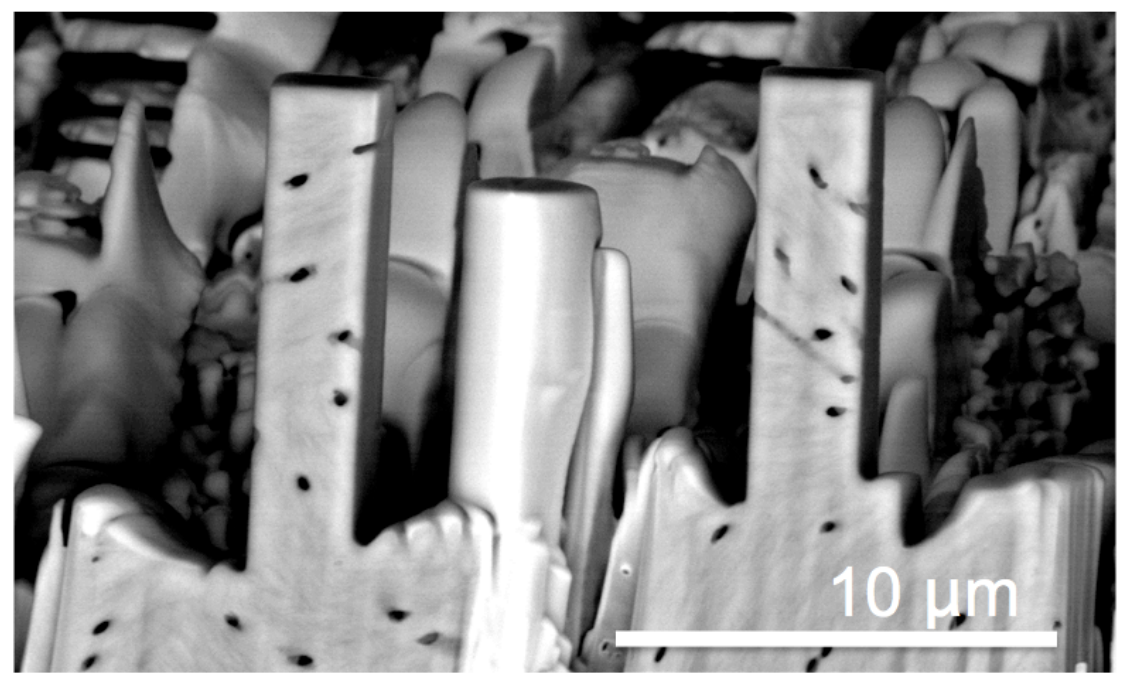

Figure 1. Electron micrograph showing a back-scattered electron image of bone microbeam samples. Porosity due to the canalicular network is observed within the micro-beams as dark regions.

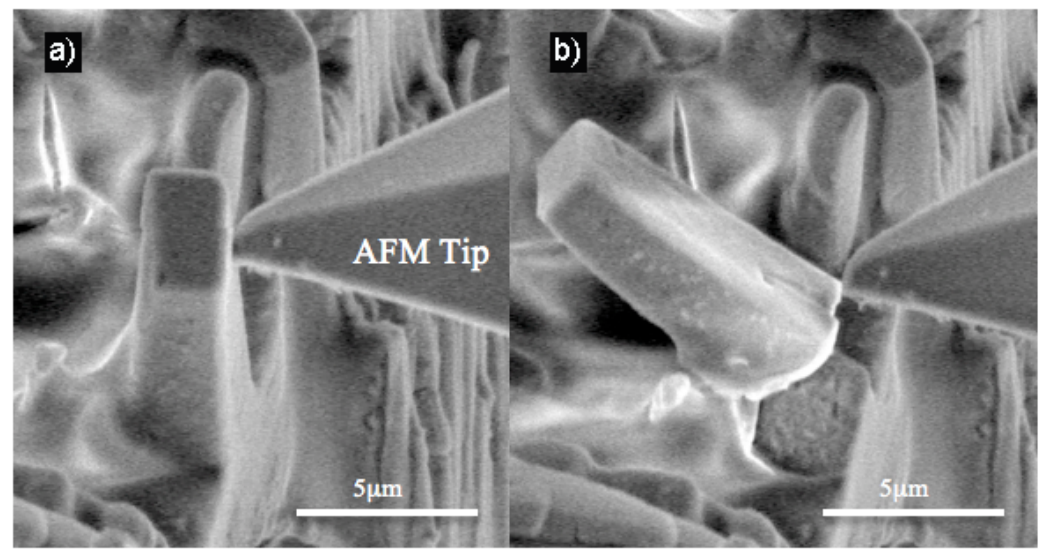

Figure 2. SEM micrographs showing a) in situ cantilever beam testing in bending provided by the AFM tip pushing into the free end of the bone micro-beam until b) failure of the micro-beam occurs. 


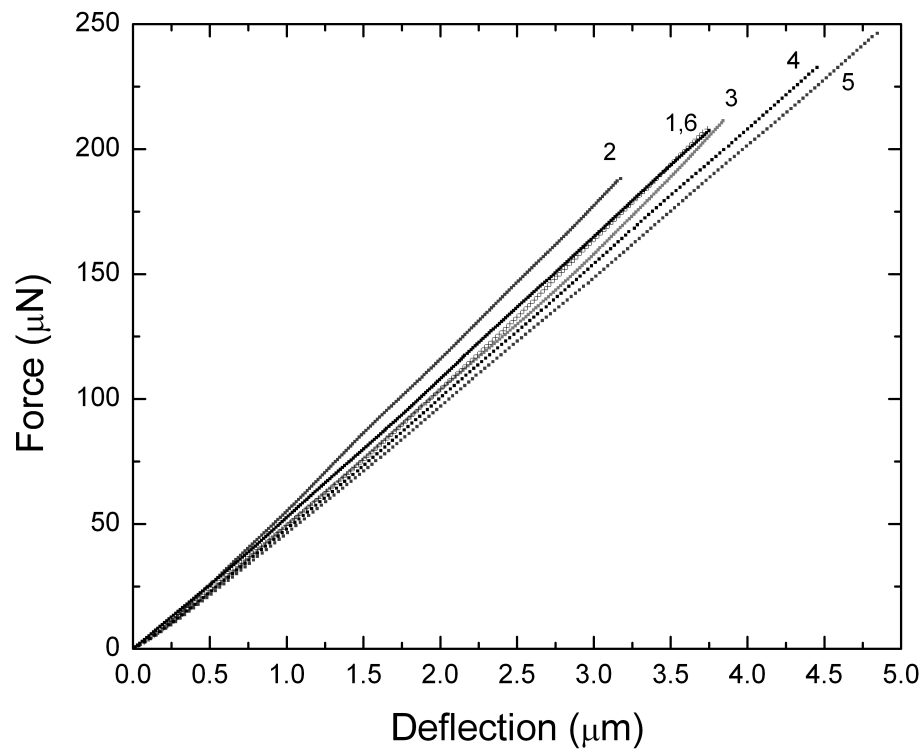

Figure 3. AFM force-deflection curves for six sub-lamellar micro-beams tested to fracture in bending. 


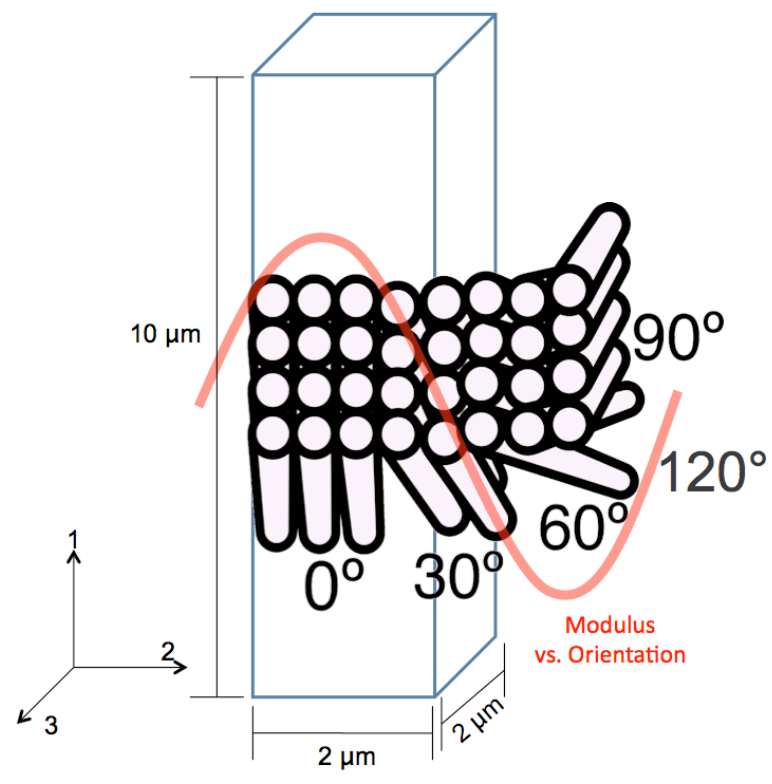

Figure 4. Schematic showing the approximate dimensions of the sub-lamellar unit in bone material produced using FIB techniques. The FIB milled bone micro-beam width is $2 \mu \mathrm{m}$, which is below the width of a single lamellar unit, thus indicating that the testing is sublamellar. In the diagram, the micro-beam contains part of the lamellar unit with the collagen fibril orientation predominantly along the long axis of the micro-beam, which will result in an elastic modulus maximum for the micro-beam. 

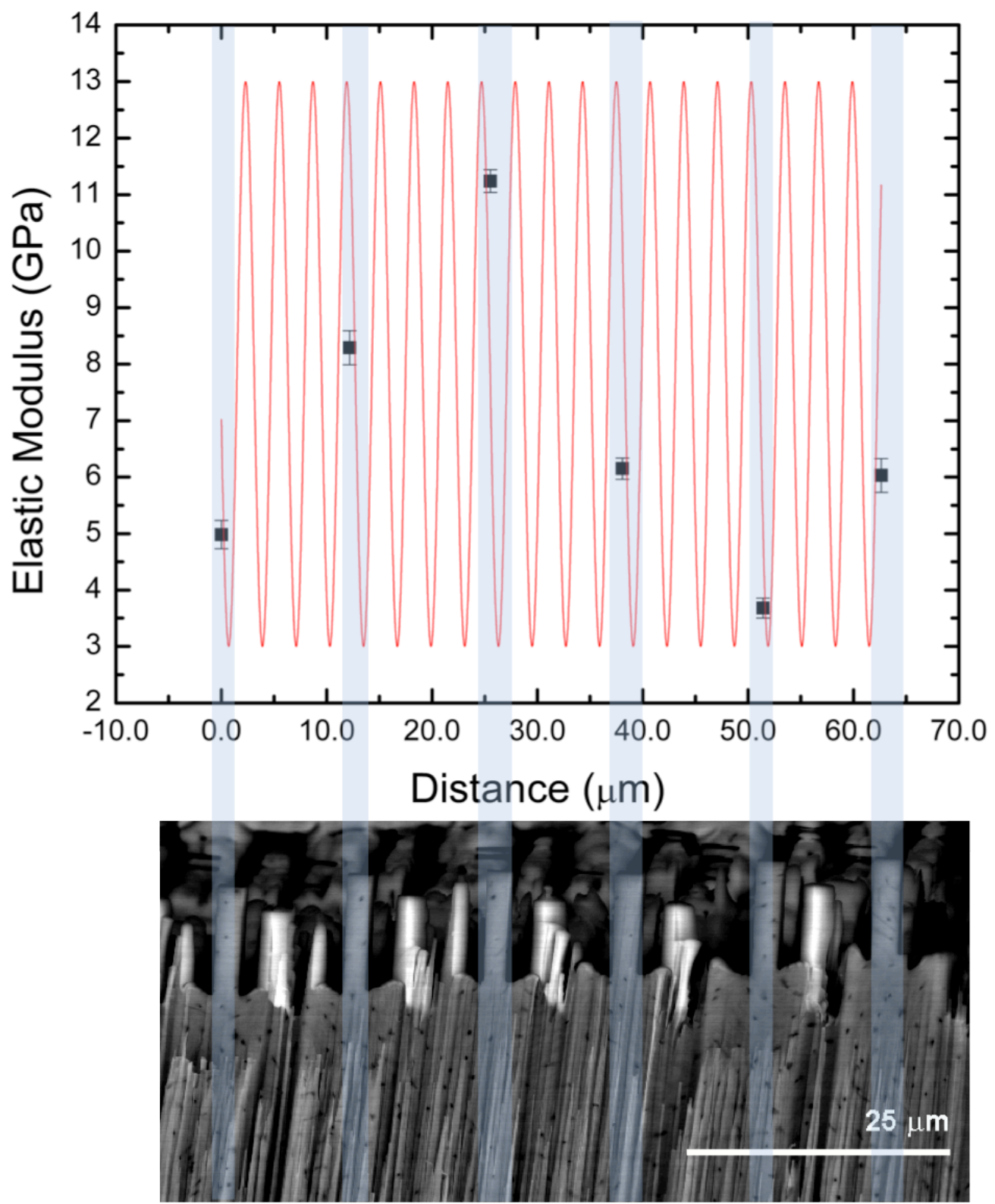

Figure 5. Plot of the variation in bone micro-beam elastic modulus against beam position (top) at the edge of the bone sample shown in the SEM micrograph (bottom). 


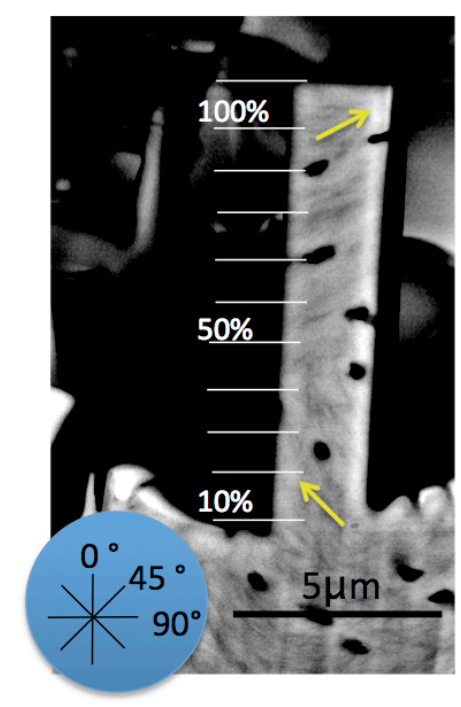

Figure 6. SEM micrograph of an individual FIB polished bone micro-beam with markers indicating the percentage distance along the beam where the fibril orientation angle was measured. Arrows highlight the observed orientation angle.

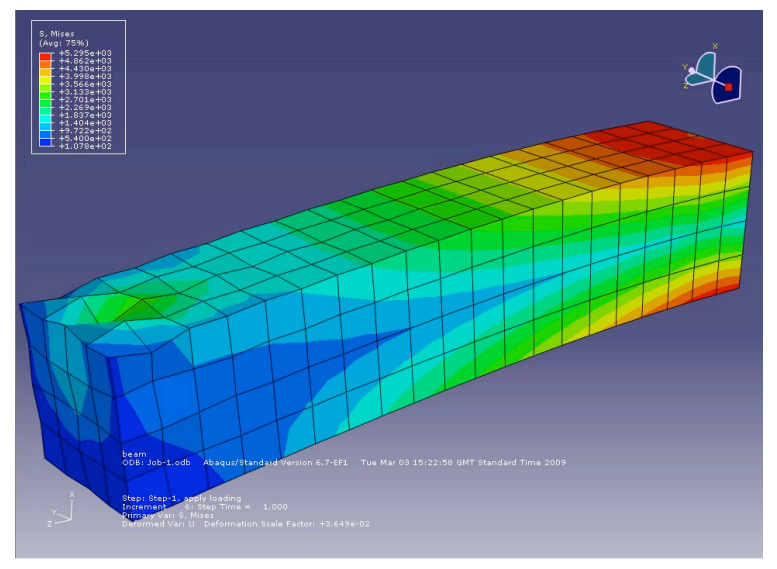

Figure 7. FEA modelling of a cantilever beam in bending, fixed at the right of the image, with a concentrated load applied to the beam free end at the left of the image. Red colouration corresponds to higher stresses while the blue corresponds to the lowest stresses. 


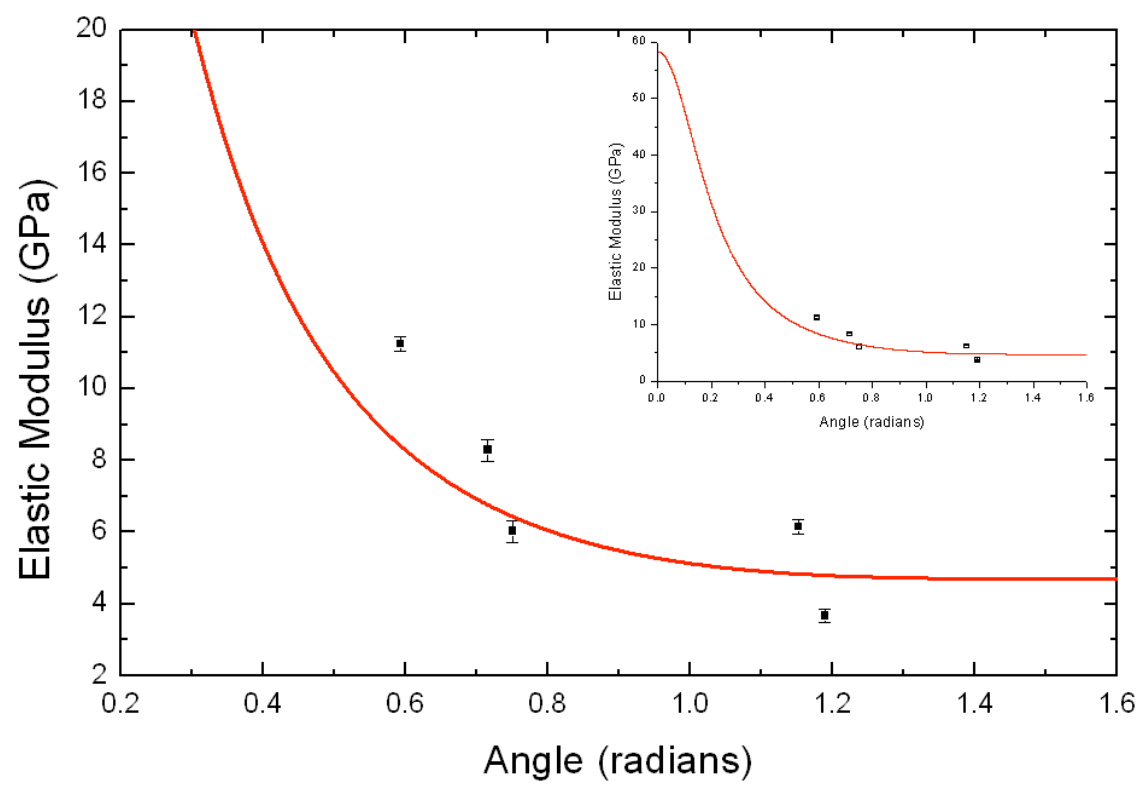

Figure 8. Plot of the variation of bone micro-beam elastic modulus with collagen fibril orientation angle taken from SEM images as demonstrated in Figure 6. A fit line from Equation (6) is provided. 

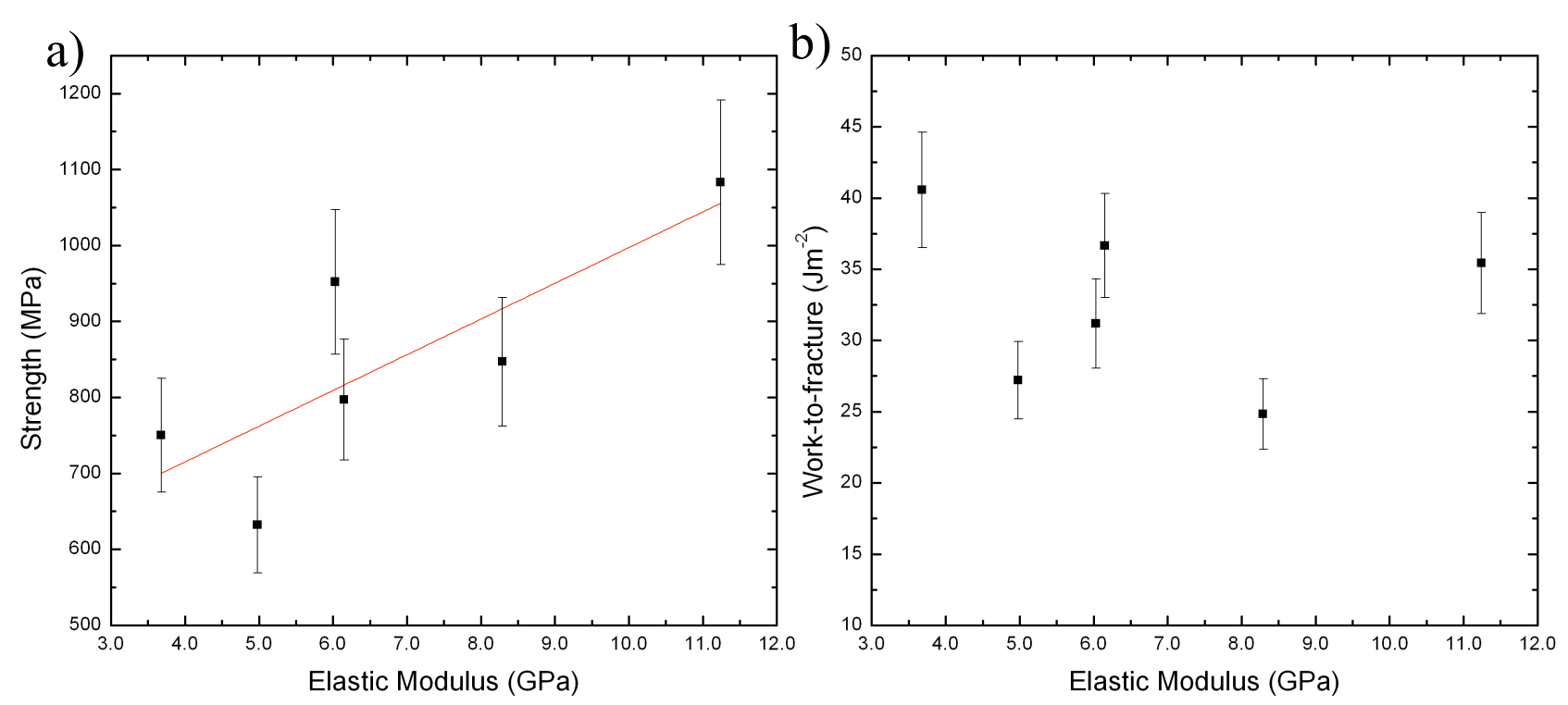

Figure 9. Plot of a) the strength of bone micro-beams against elastic modulus, showing a general increasing modulus with increasing strength $\left(R^{2}=0.6\right)$ and $\left.b\right)$ no discernable trend in work-to-fracture with increasing elastic modulus. 